\title{
Globe
}

Revue internationale d'études québécoises

\section{Matthew Evangelista, Gender, Nationalism, and War : Conflict on the Movie Screen, Cambridge, Cambridge University Press, 2011}

\section{Olivier Barsalou}

Volume 15, numéro 1-2, 2012

URI : https://id.erudit.org/iderudit/1014647ar

DOI : https://doi.org/10.7202/1014647ar

Aller au sommaire du numéro

Éditeur(s)

Globe, Revue internationale d'études québécoises

ISSN

1481-5869 (imprimé)

1923-8231 (numérique)

Découvrir la revue

Citer ce compte rendu

Barsalou, O. (2012). Compte rendu de [Matthew Evangelista, Gender, Nationalism, and War : Conflict on the Movie Screen, Cambridge, Cambridge University Press, 2011]. Globe, 15(1-2), 363-367.

https://doi.org/10.7202/1014647ar d'utilisation que vous pouvez consulter en ligne.

https://apropos.erudit.org/fr/usagers/politique-dutilisation/ 
pas reprise ailleurs qu'en introduction, notamment en raison de l'absence malheureuse d'une conclusion générale qui aurait permis de fédérer tous les chapitres en prolongeant les conclusions partielles obtenues dans chaque partie de l'ouvrage. La dernière section - à mon avis la plus convaincante -, où il est montré que la langue et son usage sont considérés comme un des seuls lieux possibles pour le sujet, contrairement au monde "physique", de s'affirmer et de se construire comme tel, aurait pu servir à confirmer avec force les intuitions du départ, à faire un tout de cette collection d'articles.

On peut également se demander si le pari de laisser de côté toute considération sociologique a été profitable pour une étude consacrée à des auteurs provenant de pays divers où la place du sujet et de l'individu parmi ses semblables, l'expérience de sa présence - et je pense surtout ici à la question du sujet féminin -, est en partie déterminée par des codes sociaux et moraux qui influencent évidemment la perception que le sujet a de luimême et de sa place dans son champ de présence. Quelques erreurs d'interprétation - notamment en ce qui a trait au vocabulaire québécois (voir l'étude sur Soucy) - ternissent également la qualité d'un ouvrage qui propose néanmoins une manière intéressante et inédite, à ma connaissance, d'aborder la construction discursive du sujet sensible en régime littéraire.

Daniel Letendre

Université de Montréal

\section{Matthew Evangelista \\ Gender, Nationalism, and War: Conflict on the Movie Screen, Cambridge, Cambridge University Press, 2011.}

Dans un essai publié en 1938, l'écrivaine américaine Virginia Woolf arguait que " as a woman I have no country. As a woman I want no country. As a woman my country is the whole world ${ }^{1} »$. Elle rejetait toute forme de patriotisme féminin, car le contrat social liant l'État et ses citoyens exclut les femmes. L'ouvrage de Matthew Evangelista, professeur en science politique à l'Université Cornell (États-Unis), prend pour point de départ cette présomption selon laquelle les femmes sont simplement les victimes des

$$
\div+\div
$$

1. Virginia WoOLF, The Three Guineas, Londres, Hogarth Press, 1938. 
conflits nationalistes. Ce truisme obscurcit le rôle passif ou actif, souvent privé, que jouent les femmes dans la production et la reproduction de la nation ${ }^{2}$.

Woolf établit des liens entre les violences nationalistes et la féminité. Selon elle, ce sont les institutions de la socialisation (école, religion, appareil d'État) qui, de par leur nature hiérarchique et compétitive, inculquent le désir guerrier et nationaliste chez les hommes. Le statut dit "privé» de la femme dans la société est la résultante logique d'un processus de socialisation/intégration duquel elles sont exclues. Cette exclusion des institutions masculines serait, selon Woolf, la source du pacifisme des femmes.

Le professeur Evangelista explore ces thèmes et cherche à problématiser les hypothèses de Woolf au travers de l'analyse de films issus de quatre régions du monde qui ont été le théâtre de conflits nationalistes : la guerre d'indépendance d'Algérie, le démembrement de la Yougoslavie, la guerre en Tchétchénie et le nationalisme québécois. À l'instar de la littérature, le cinéma, matériau sous-utilisé en sciences sociales, réussit souvent à mettre en lumière, de façon subtile et sensible, les impensés de la relation complexe liant le genre, le nationalisme et la guerre.

Deux grandes hypothèses de recherche se dégagent du livre. La première, inspirée par la pensée de Woolf, suggère que l'amélioration relative du statut de la femme vis-à-vis de l'homme tend à réduire les conflits entre communautés politiques. La seconde, empruntée aux théories associées, entre autres, à Cynthia Enloe ${ }^{3}$, suggère qu'un déclin dans le statut et les perspectives économiques des hommes, dans la mesure où cela affecte négativement leur identité masculine, peut conduire à des éruptions de violence qui s'exprimeront tant à l'intérieur de la communauté, contre les femmes par exemple, qu'à l'extérieur de celle-ci. Cet écroulement des identités masculines conduirait à une "prolifération de petits hommes ${ }^{4}$. Leur déclin économique ne correspondrait pas aux attentes sociales et à l'image traditionnelle d'une masculinité forte et confiante. Dans ce contexte, la violence s'exprimerait à la fois contre l'oppresseur étranger à la communauté ainsi que contre les femmes, dont l'émancipation continue de pervertir l'image qu'a la société des hommes.

$$
+\div
$$

2. Nira YUVAL-DAVIS, "Les femmes et le nationalisme", Les Cahiers du Grif, n 48, 1994, p. 89-96.

3. Cynthia ENLOE, Bananas, Beaches and Bases: Making Feminist Sense of International Politics, Nouvelle édition revue et augmentée, Berkley, University of California Press, 2000.

4. Matthew Evangelista, Gender, Nationalism, and War: Conflict on the Movie Screen, Cambridge, Cambridge University Press, 2011, p. 14. 
Par exemple, le film La Bataille d'Alger (1966), mis en scène par le réalisateur italien Gillo Pontecorvo, évoque avec beaucoup de réalisme l'ambiguïté du rôle de la femme dans la guerre de libération nationale algérienne et réfute du même coup la thèse de Woolf. En effet, ce film met en scène des femmes participant volontairement aux actes de violence du Front de libération nationale (FLN) algérien, que ce soit en posant des bombes ou en trafiquant des armes à l'intérieur de la casbah d'Alger. En ex-Yougoslavie, le film Joli village, jolie flamme du réalisateur Srdjan Dragojevic raconte avec ironie la montée de ces tensions intercommunales et l'absurdité de la guerre. Surtout, comme le décrit Evangelista, les violences envers les femmes se sont nourries de la culture, d'un vocabulaire et d'un sentiment serbes profondément misogynes. Les conflits nationalistes dans les Balkans rompent définitivement, selon Evangelista, avec l'image pacifique de la femme et guerrière de l'homme. L'auteur évoque plusieurs cas où des femmes ont choisi de joindre les rangs de l'armée afin de combattre le nationalisme serbe et ainsi préserver le caractère multiethnique de leur communauté. La situation en Tchétchénie, république caucasienne de la Fédération de Russie, est, quant à elle, plus près de celle qui prévalait en Algérie que du cas yougoslave. Dans ce chapitre, Evangelista revisite plusieurs films, surtout russes, sortis dans les années 1990 et $2000^{5}$. Ces films évoquent la coopération entre femmes (mères, femmes de soldats, etc.) russes et tchétchènes, et ce, en dépit des différences ethniques et nationales qui les séparent. Bien que les films demeurent ambigus sur le pacifisme réel de ces femmes, il n'en demeure pas moins qu'ils valident, en surface du moins, l'hypothèse de Woolf.

La dernière étude de cas est consacrée au nationalisme québécois, pour lequel Matthew Evangelista semble éprouver une certaine sympathie, tel que dépeint par Robert Lepage dans le film Nô sorti en 1998. Contrairement aux conflits algérien, yougoslave et tchétchène, la bataille nationaliste au Québec est livrée pacifiquement. Est-ce le rôle important des femmes dans la société québécoise qui explique le caractère non violent, voire pacifique, du conflit nationaliste québécois ? Evangelista et Lepage répondent par l'affirmative'.

La crise d'Octobre de 1970 a brouillé les rapports entre violence, genre et nationalisme au Québec. Cet événement est aussi le point de départ

$$
4+4
$$

5. Le prisonnier du Caucase, Sergueï Bodrov, réalisateur, 1996, 98 min ; Checkpoint, Aleksandr Rogozhkin, réalisateur, 1999, 85 min ; Voyna (War), Alekseï Balabanov, réalisateur, 2002, 120 min ; Caucasian Roulette, Fyodor Popov, réalisateur, 2002, 83 min.

6. Michel CoulOMBE, «Entretien avec Robert Lepage», Ciné-Bulles, vol. 14, n 4, 1995, p. 20-24. 
du film $N o ̂$ de Lepage. Le $N o ̂$ est une forme théâtrale traditionnelle japonaise. L'expression, anglophone de surcroît!, réfère aussi à la réponse des Québécois au référendum de 1980. Le scénario du film lie le personnel au politique et illustre la vision de Lepage d'un Québec matriarcal. Dans ce film, les hommes se battent pour la langue et la culture avec violence et un certain ridicule théâtral. Les femmes, représentées par Sophie, interprétée par AnneMarie Cadieux, se battent, quant à elles, pour se réapproprier leur corps qui est aussi le véhicule de la reproduction et de la survie de la nation et de la culture québécoises.

Au travers de la grossesse non désirée de Sophie, Lepage illustre à la fois les violences faites aux femmes du Québec des années 1970 et les problèmes du nationalisme québécois. Au plan personnel, Sophie, alors qu'elle se trouve au Japon, se demande comment elle pourrait se faire avorter dans le Québec des années 1970 alors que ce droit n'est toujours pas reconnu ${ }^{7}$. Au plan politique, la grossesse de Sophie illustre l'enjeu auquel feront face les Québécois en 1980 : la possibilité de donner naissance à un nouveau pays. À son retour chez elle, au Québec, Sophie est arrêtée et brutalisée par les policiers qui surveillaient son conjoint, interprété par Alexis Martin, sympathisant du Front de libération du Québec (FLQ). La violence des policiers provoque une fausse couche chez Sophie. Le sang qui coule le long de sa jambe est l'illustration forte de la condition politique du Québec et de celui des femmes à une époque marquée par des violences nationalistes. Cette fausse couche illustre aussi l'échec de la stratégie violente du FLQ.

La lecture subtile et très adroite que fait Evangelista, probablement la plus aboutie du livre, démontre bien les points de rupture et de convergence entre nationalisme, violence et genre. Cette lecture illustre aussi la condition politique des femmes du Québec au cours de cette période ${ }^{8}$. Celles-ci demeurent à la fois les sujets et les objets du nationalisme. D'un côté, elles se battent pour l'obtention d'une plus grande autonomie dans la sphère publique, alors que de l'autre, elles demeurent l'objet de l'intervention du mouvement nationaliste, car leur corps demeure le véhicule de la reproduction et de la transmission de la culture. Les politiques natalistes québécoises des 30 dernières années témoignent de cette ambiguïté.

$$
4 \div 4
$$

7. Sauf dans les cas de femmes en danger. Le droit à l'avortement ne fut reconnu qu'en 1988 .

8. Sur ce point, voir l'article de Sean MILLS, "Québécoises deboutte! Le Front de libération des femmes du Québec, le Centre des femmes et le nationalisme", Mens: Revue d'histoire intellectuelle et culturelle, vol. 4, n 2, 2004, p. 183-210. Le cri de ralliement du Front de libération des femmes du Québec (FLF), crée à la fin des années 1960, était "pas de libération du Québec sans libération des femmes, pas de libération des femmes sans libération du Québec». 
Dans ce contexte, comment le Québec est-il parvenu à éviter les violences nationalistes qui ont déchiré la Yougoslavie? Evangelista identifie trois raisons. La première est qu'il y a une plus grande égalité entre les sexes au Québec et moins de barrières à l'emploi tant pour les hommes que pour les femmes. La seconde explication se trouve dans le fait que le potentiel de violence nationaliste contre les femmes demeure bas, bien que le problème de la violence contre les femmes demeure toujours préoccupant, comme le massacre de la Polytechnique en décembre 1989 nous le rappelle. La dernière explication se trouve dans la montée du multiculturalisme au Québec. Cette idée témoigne du fait que les identités sont polymorphes et plastiques: elles peuvent être non violentes, plurielles et non exclusives. Toutefois, les débats sur les libertés religieuses et le droit des femmes qui animent le discours politique québécois actuel (et passé) illustrent de façon éloquente que la femme, en tant que site du pouvoir masculin, demeure au cœur des préoccupations tant multiculturalistes que nationalistes. De ce point de vue, le féminisme de Woolf, affranchi de ses repères masculins, semble retrouver toute sa pertinence et sa fraîcheur (voire sa nécessité) dans ce contexte québécois marqué par la résurgence d'une pensée conservatrice critiquant l'héritage de la Révolution tranquille, l'idéologie des droits de la personne ainsi que les repères classiques de la modernité québécoise $e^{9}$.

L'ouvrage de Matthew Evangelista, sans être révolutionnaire, fait la démonstration, au travers de présentations schématiques nuancées, des qualités heuristiques et pédagogiques $\mathrm{du} 7^{\mathrm{e}}$ art. Celui-ci peut nous aider à mettre en lumière les ambiguïtés et paradoxes de notre propre condition politique, chose que les sciences humaines classiques peinent à rendre compte dans toute leur complexité. Le livre de Matthew Evangelista nous rappelle aussi que les rapports entre violence, genre et projets nationalistes, quels qu'ils soient, demeurent équivoques et éminemment ambigus, et ce, tant pour les hommes que pour les femmes. Toute généralisation sur le sujet se révèle donc vaine, en dépit des similarités qui pourraient exister. De ce point de vue, cet ouvrage constitue un effort réflexif extrêmement utile.

Olivier Barsalou New York University

$$
4 \div
$$

9. Voir le numéro intitulé «Qu'est-ce qu’être conservateur» de la revue Argument, vol. 14, nº 1, Automne 2011/Hiver 2012 et Chedly BELKHODJA, "Le discours de la "nouvelle sensibilité conservatrice" au Québec", Études ethniques au Canada, vol. 40, n 2, 2008, p. 79-100. Voir aussi Rima ELKOURI, "Les chauffards de la laïcité ", La Presse, 14 mars 2012, p. A21. 